\title{
ECONOMÍA DEL CONOCIMIENTO, CRISIS FINANCIERA Y DEPRESIÓN
}

\author{
Ugo Pagano* \\ Maria Alessandra Rossi***
}

T a economía del conocimiento se suele invocar como clave de Lprogreso, desarrollo y prosperidad. Desde la obra de Schumpeter (1934 y 1942), la producción de conocimiento y la innovación se consideran características de las economías de mercado, esenciales para superar la inercia social y, como después plantearon Abramovitz (1959) y Solow (1960), más relevantes que la acumulación de capital para explicar el crecimiento. Una rama reciente de investigación subraya, sin embargo, que las actuales instituciones de la economía del conocimiento, lejos de ser motores infalibles del crecimiento económico, poseen características que pueden llevar a su propia desaparición $\mathrm{y}$ al estancamiento del crecimiento.

La clave para entender por qué el final de la economía del conocimiento puede ser la crisis y la depresión es el análisis de la dinámica que lleva a reducir las oportunidades de inversión debido al escalamiento de los vallados de conocimiento asociados al fortalecimiento del sistema de propiedad intelectual (PI) y al debilitamiento de las instituciones de la "ciencia abierta". La creciente monopolización de los recursos intelectuales produce efectos de retroalimentación virtuosos y viciosos entre distribución de los activos intelectuales e incentivos para aprender y desarrollar nuevo conocimiento. $\mathrm{Y}$ aun

*Universidad Central Europea y Universidad de Siena, Italia, [ugo.pagano@ unisi.it].

** Universidad de Siena, Italia, [alessandra.rossi@unisi.it]. Working paper publicado en Quaderni del Dipartimento di Economia Politica e Statistica 741, Universidad de Siena, diciembre de 2016. Traducción de Alberto Supelano. Fecha de recepción: 16-12-2016, fecha de revisión: 15-02-2017, fecha de aceptación: 27-04-2017. Sugerencia de citación: Pagano, U y M. A. Rossi. "Economía del conocimiento, crisis y depresión", Revista de Economía Institucional 19, 36, 2017, pp. 57-74. Dor: https://doi.org/10.18601/01245996.v19n36.03 
donde operan ciclos virtuosos, cuanto más se reduce la participación del conocimiento no privatizado en favor de monopolios intelectuales, menos son las oportunidades de inversión globales y, por tanto, menos capaz es la economía del conocimiento de mantener sus promesas de crecimiento.

La reducción de la proporción del conocimiento público disponible es agravada por la economía política de la protección de la pi y de la financiación pública de ciencia abierta. A nivel nacional, las actividades de caza de rentas de las grandes firmas y la captura de quienes toman tales decisiones explican muchos aspectos de la evolución de los sistemas nacionales de PI y de las políticas de innovación. Pero este no es el final de la historia. A nivel internacional intervienen muchas fuerzas que intentan ampliar los vallados de conocimiento. De hecho, cuando las reglas de reciprocidad entran en vigencia en el dominio internacional de la PI aumentan los incentivos de los países para armonizar (hacia arriba) sus normas de PI y como resultado se tiende a sobreproteger la PI. Más en general, el carácter global común de los recursos de conocimiento abre espacio al comportamiento de gorrón por el cual cada país tiene un incentivo para usar el conocimiento público de otros países y privatizar en exceso el conocimiento que produce. A nivel nacional e internacional el problema es agravado por efectos de retroalimentación ubicuos: una vez operan las instituciones de PI, las firmas (países) se encuentran en una situación de dilema del prisionero en la cual el patentamiento (el fortalecimiento de la protección de patentes y la reducción del ámbito del conocimiento público disponible) es una estrategia dominante para todos, aunque una estrategia de mayor apertura concordaría con la maximización conjunta del bienestar.

En este artículo planteamos que la existencia de estas fuerzas endógenas, con características que se refuerzan a sí mismas, debería ser una nueva justificación y un fundamento de la política científica, en particular de una política científica global. Puesto que no hay un antídoto espontáneo contra la creciente tendencia a la excesiva privatización del conocimiento, se necesitan con urgencia políticas que reconozcan expresamente los riesgos inherentes a esta privatización excesiva. Además, se requieren esfuerzos de coordinación internacional con el fin de evitar la distorsión de los incentivos para invertir en investigación pública debido a las externalidades transfronterizas no compensadas. Nuestra perspectiva no solo sugiere que se deben abandonar los prejuicios neoliberales contra la inversión pública directa en investigación, sino que la financiación de investigación pública no se 
debe considerar en forma aislada de la manera apropiada de difundir sus resultados. Sin políticas orientadas a corregir el balance entre conocimiento privado y conocimiento público disponible es difícil que la economía del conocimiento cumpla su promesa de mejorar el crecimiento.

La primera sección ilustra las razones que explican por qué el final de la economía del conocimiento puede ser la crisis y la depresión. La segunda sección trata el tema de la economía política de los vallados de conocimiento. La tercera sección presenta la justificación de una nueva política científica (global). La última resume las principales preguntas de futura investigación.

\section{¿POR QUÉ EL FINAL DE LA ECONOMÍA DEL CONOCIMIENTO PUEDE SER LA CRISIS Y LA DEPRESIÓN?}

En las últimas décadas, la fe en las características de la economía del conocimiento que propician el crecimiento y en los pilares subyacentes de investigación e innovación científica y tecnológica ha ido acompañada de la creencia, igualmente general, en que instituciones afines a la propiedad privada pueden dar en el campo de los intangibles el mismo tipo de beneficios que proporcionan en el ámbito tangible. Esta concepción ha despertado los intereses más mundanos de las grandes empresas del mundo desarrollado y llevado a un fortalecimiento sin precedentes de la protección de la PI a nivel global.

La intensa privatización de los recursos intelectuales también ha sido impulsada por extraordinarios desarrollos tecnológicos que, por un lado, subrayan la extensión del material patentable y, por el otro, aumentan las posibilidades globales de imitar y copiar invenciones y creaciones intelectuales. Los avances en las tecnologías de la información y la comunicación (TIC) y la creciente complejidad de las interacciones entre disciplinas científicas (p. ej., en nano materiales, bioinformática, etc.) están desdibujando las una vez claras distinciones entre ciencia básica y aplicada, llevando así a una notable ampliación del "cuadrante de Pasteur" (Stokes, 1997), es decir, del área de la investigación científica que es a la vez básica y aplicada. Productos, procesos de producción e industrias enteras se caracterizan por una complejidad cada vez mayor y se basan en innovaciones entrelazadas y acumulativas, asociadas tanto a resultados previos de la investigación básica y aplicada como a desarrollos tecnológicos paralelos. Al desdibujarse las líneas entre el campo de la tecnología y el de la ciencia pura y rechazarse el modelo lineal de innovación, el ámbito del material patentable ha crecido notablemente. Al mismo 
tiempo, la difusión global de las Tic ha ampliado el alcance global del conocimiento técnico y de las innovaciones, a la vez que se expande el margen para su apropiación indebida.

Estos desarrollos científicos y tecnológicos sugieren algunas de las razones por las cuales la analogía entre propiedad y PI que sustenta muchos discursos de política (comercial, industrial y científica) es engañosa y peligrosa para la misma economía del conocimiento. A diferencia de la propiedad tangible, la PI implica un mayor margen de traslapo de derechos "exclusivos", lo que hace difícil identificar con exactitud al propietario de un recurso intelectual dado, y provoca conflictos costosos e improductivos y, lo que es más importante, puede obstaculizar su explotación productiva. La causa esencial son las características de bien (cuasi) público inherentes al conocimiento, que dan lugar a un desajuste entre las relaciones legales definidas por la propiedad privada y el carácter ilimitado del conocimiento y de la información como recursos productivos (Arrow, 1996, 651). La no rivalidad del conocimiento implica que la exclusión artificial de terceros asociada a la PI se genera a costa de una ineficiencia (que se suele aceptar como un mal necesario a cambio de mayores incentivos para producir conocimiento). También implica que el tamaño y la extensión potencial de esta exclusión son de un orden de magnitud no comparable con el de la propiedad privada. Como se argumenta en otro artículo de uno de los autores: "la propiedad privada absoluta del conocimiento constituye un monopolio global que limita la libertad de muchos individuos en numerosos lugares" (Pagano, 2014, 1413).

Los estudios de muchas vertientes intelectuales con diferentes agendas de investigación han empezado a reconocer estas tensiones y a resaltar las razones por las cuales la innegable tendencia a la privatización de los recursos de conocimiento puede ser excesiva desde el punto de vista social y terminar socavando el funcionamiento de los motores de producción de conocimiento.

Una primera vertiente se centra en las deficiencias de las actuales instituciones de PI, con especial atención en el sistema de patentes. Sus estudios, que suelen explorar el vínculo entre patentes e innovación, resaltan la existencia de efectos contrarios a la pretensión de que mayor patentamiento implica necesariamente mayor innovación. Muchos especialistas en derecho han expresado su escepticismo, en especial los que han estado expuestos más directamente a la mecánica real del régimen de PI (p. ej., Benkler, 2002; Lemley, 2005; Samuelson, 2006). También existe un conjunto coherente de estudios económicos que consideran que las patentes pueden tener un efecto perjudicial sobre 
la innovación (bien representados, p. ej., por Jaffe y Lerner, 2006; Bessen y Meurer, 2008; Boldrin y Levine, 2008).

Algunos trabajos muestran teóricamente que cuando la investigación es secuencial y se basa en innovaciones previas, patentes más fuertes pueden desalentar invenciones posteriores (Merges y Nelson, 1990; Scotchmer, 1991) y que el traslapo de derechos de patente puede llevar a la "tragedia de los anti comunes", un caso de subexplotación de recursos intelectuales debido a la proliferación de derechos de veto sobre su uso (Heller y Eisenberg, 1998).

Desde el punto de vista empírico, ya hace tiempo (al menos desde los años ochenta) se sabe que en muchos sectores las patentes son a lo sumo de utilidad limitada y que las firmas consideran menos efectivos los mecanismos formales de protección que sus alternativas (Mansfield, 1986; Levin et al., 1987; Cohen et al., 2000; varias ediciones de la Community Innovation Survey), aunque su propensión a patentar sigue siendo alta. Diversos estudios señalan que las firmas patentan porque otras patentan y no por la utilidad intrínseca de las patentes. Quizá opere una “paradoja de las patentes": el sistema de patentes crea incentivos para patentar y no para invertir en I y D (Hall y Ziedonis, 2001), en especial cuando las firmas refinan el uso de patentes como herramienta estratégica para impedir inversiones innovadoras de sus competidores, mejorar su posición negociadora en la concesión de licencias o defenderse de litigios sobre patentes (ver, p. ej., Arundel et al., 1995; Duguet y Kabla, 1998; Reitzig et al., 2010).

Otras investigaciones revelan las distorsiones que causan las patentes en la innovación, que inducen costosas duplicaciones de esfuerzos de investigación, trastocan las trayectorias tecnológicas de las firmas, las alejan de áreas con mayor riesgo de infracción de la PI de terceros y desalientan el inicio de los proyectos innovadores que tienen mayor probabilidad de causar problemas por traslapos de patentes. Estos problemas se agravan en áreas donde los productos son tecnológicamente complejos y los portafolios de patentes de las firmas pueden alcanzar una escala sustancial; un problema al que se suele llamar "maraña de patentes"1.

Lerner (1995) presenta evidencia de que las firmas nuevas y pequeñas de biotecnología con altos costos de litigio se abstienen de patentar en áreas donde hay mayor probabilidad de infringir patentes existentes, en particular donde la propiedad pertenece a firmas (grandes) con bajos costos de litigio. Cockburn et al. (2010) demuestran

\footnotetext{
${ }^{1}$ Shapiro (2001) llama "maraña de patentes" a las densas redes de derechos de patente traslapados, en su mayoría pertenecientes a portafolios de grandes firmas.
} 
que la necesidad de otorgar licencias de patente reduce el desempeño innovador de las firmas, con base en una encuesta a firmas alemanas innovadoras. Noel y Schankerman (2013), en un estudio sobre patentamiento en la industria de programas de computador entre 1980 y 1999, encuentran que las compañías que enfrentan alta concentración de portafolios de patentes entre sus principales rivales se abstienen de invertir en I y $\mathrm{D}$ en áreas donde los portafolios de patentes de sus rivales son más fuertes.

Un campo contiguo de investigación es el del patentamiento académico. En Estados Unidos la Ley Bayh-Dole de 1980 permitió patentar resultados de investigación financiada por el gobierno federal, y abrió el camino a la creciente privatización de la ciencia financiada públicamente que se extendió a muchos países de la OCDE. Ese cambio en la política científica se justificó por varias razones: facilitar la comercialización, contrarrestar el efecto de la reducción de financiación pública y, más en general, reorientar la investigación académica y de las organizaciones de investigación públicas (OIP) a campos que contribuyan a cumplir la promesa de la economía del conocimiento de propiciar el crecimiento. A ese respecto, la investigación se ha centrado en un doble vínculo: entre patentamiento y velocidad del avance científico, y entre herramientas de difusión y resultados de la investigación (para una revisión concisa, ver Franzoni y Scellato, 2011).

Para el propósito de este artículo son relevantes tres resultados de esa literatura. Primero, las universidades y las oip no son muy buenas en el juego del patentamiento, bien se mire la proporción de sus patentes en el total (un 5\% de las patentes activas en Estados Unidos según Thursby y Thursby, 2007, y una cifra similar en Europa según Lissoni et al., 2008) o los ingresos que obtienen (Geuna y Nesta, 2006). Además de suscitar dudas sobre la eficacia del patentamiento académico para promover la comercialización, esto debería bastar para preguntar si, desde el punto de vista de las oip, los posibles beneficios de las patentes son mayores que la restricción de la libertad de investigación causada por el recorte de la exención a la investigación ${ }^{2}$.

${ }^{2}$ En Estados Unidos, la sentencia clave que sanciona el recorte de la exención a la investigación de las universidades es la decisión del Tribunal Federal del Circuito de Apelaciones en el caso Madey v. Duke University (307 F.3d 1351, 1362, Fed. Cir., 2002). En esa sentencia, el Tribunal sostuvo que la exención "no se aplica a actividades realizadas en el curso del 'negocio' normal de una institución de investigación, con o sin ánimo de lucro”. Es claro que cuantas más oip se dediquen a patentar, tanto más sus actividades se considerarán parte del negocio, que no merecen exención. En Europa, los actos "realizados en privado y con fines no comerciales" y los actos "realizados con fines experimentales relacionados con el objeto de la invención" tradicionalmente han sido protegidos de la obligación. 
Segundo, hay evidencias de que el patentamiento académico impide difundir los resultados de la investigación. Un artículo muy interesante examina el "experimento natural" de llevar al dominio público patentes relacionadas con un ratón diseñado genéticamente (Murray et al., 2009). Los autores encuentran que la investigación en el área aumenta notablemente y es más diversificada por la apertura de nuevas vías de investigación que no se siguen cuando operan las patentes. Con una metodología diferente, Franzoni y Scellato (2010) encuentran rezagos significativos en la publicación en revistas científicas cuando los resultados son patentados. Y Campbell et al. (2002) y Walsh et al. (2007) encuentran evidencia de que se oculta información, datos y materiales en los que se basa la investigación.

Tercero, poco se puede decir del efecto del patentamiento en la velocidad del avance científico. Aunque no parece haber trade-offs simples entre publicar y patentar si se mira la productividad de cada investigador (p. ej., Azoulay et al., 2009), no existen trabajos que aborden el asunto clave: ¿las externalidades negativas para otros investigadores del mismo campo están asociadas a las patentes? (Franzoni y Scellato, 2011).

Mientras que los trabajos mencionados hasta ahora se centran en los vínculos causales directos entre patentamiento e innovación o entre patentamiento y avance científico, hemos propuesto en trabajos anteriores que los vínculos entre derechos de propiedad intelectual (DPI) e inversiones innovadoras se auto refuerzan, y que ese es el origen tanto de los patrones de desigual distribución de los recursos intelectuales y de las oportunidades de crecimiento como de la progresiva reducción global de las oportunidades de inversión. Esto a su vez indica la existencia de un mecanismo endógeno a la economía del conocimiento que puede ser parte de la explicación de su crisis.

El punto de partida para reconocer las relaciones de auto reforzamiento existentes en el campo de la PI es la diferencia ya mencionada entre propiedad de bienes tangibles y propiedad intelectual. E1 argumento de eficiencia clave que subyace a la existencia de instituciones de propiedad privada está asociado al efecto del incentivo que puede generar la propiedad. Los propietarios tienen incentivos para mantener, mejorar y usar productivamente su propiedad tangible. Y, más importante, para invertir en capital humano específico de valor creciente, como reconocen los exponentes del nuevo enfoque de los derechos de propiedad (p. ej., Hart, 1995). Pagano y Rossi (2004)

Pero está por ver si las actividades de las or peguirán protegidas cuando aumenten el patentamiento y los traslapos entre investigación pública y privada. 
resaltan que este efecto es mucho más fuerte para la pi que para la propiedad física, porque quienes poseen PI tienen un derecho a excluir de mayor alcance, pues esta restringe la libertad de terceros a replicar medios de producción similares. Esto, a su vez, origina grandes efectos de retroalimentación: mientras que los propietarios tienen fuertes incentivos para invertir en aprendizaje y capital humano específico de PI y adquirir activos intelectuales, los no propietarios son desincentivados para invertir en adquisición de capital intelectual. Se generan círculos virtuosos de acumulación y círculos viciosos de exclusión del capital intelectual, con evidentes propiedades de auto reforzamiento.

Esta perspectiva da un paso más allá de los análisis mencionados en cuanto destaca los efectos del tamaño de la firma y del portafolio de patentes sobre la inversión en I y D y los patrones de patentamiento, y resalta su carácter auto reforzador a nivel de firmas y de países. A nivel de la firma, la creciente privatización del conocimiento impide desarrollar formas de organización de la producción más democráticas que las que parece permitir el mayor contenido de conocimiento de la producción actual (Pagano y Rossi, 2011; Pagano, 2014). Cuando existen derechos de monopolio y se puede codificar gran parte del conocimiento relevante para la producción, las firmas capitalistas gozan de una ventaja de costos con respecto a las firmas poseídas por trabajadores porque su tamaño y la exclusión artificial inducida por la PI alimentan en forma dramática los rendimientos crecientes (restringidos artificialmente a nivel de la firma).

Los efectos auto reforzadores de la PI son aún más profundos a nivel de país. La dotación de DPI tiende a estar en el origen de nuevas formas de ventaja comparativa (Belloc y Pagano, 2012): puesto que los DPI son globales, los países encuentran obstáculos para especializarse en productos que dependen fuertemente del conocimiento protegido por la PI de otros países. Esto da lugar a patrones de especialización forzada que alimentan el comercio global, y son una causa novedosa de un mayor intercambio internacional, además de las explicaciones clásicas de la teoría del comercio. Lo más relevante es que estos patrones de especialización forzada tienden a perpetuarse en el tiempo, y así dan lugar a trayectorias de desarrollo y subdesarrollo asociadas a la desigual distribución inicial de dotaciones de PI.

Pagano y Rossi (2009) y Pagano (2014) argumentan que estos efectos de retroalimentación afectan no solo las pérdidas y ganancias relativas de países asimétricamente dotados sino también las oportunidades globales de inversión y, así, el crecimiento. El efecto total del fortalecimiento global de la protección de la PI puede haber sido, 
de hecho, una contracción global de las oportunidades de inversión productiva, que puede ser la base de la crisis reciente. La falta de buenas oportunidades de inversión, junto a la abundancia de capital y las laxas regulaciones financieras, puede explicar por qué el capital se retiró del uso productivo y así dio lugar a la burbuja inmobiliaria y a la crisis subprime posterior (Pagano y Rossi, 2009). Además, la privatización del conocimiento contribuye a la financiarización de la economía, pues convierte los recursos intelectuales en activos de propiedad seguros y transables que, sin un valor definido en un mercado competitivo, están fácilmente expuestos a los caprichos de expectativas especulativas (Pagano, 2014).

La evolución de la inversión global parece concordar con la idea de que los DPI globales reducen gradualmente las oportunidades de inversión. Como se ve en la gráfica 1 , después de la medida que desencadenó el fortalecimiento global de los DPI (el Acuerdo de 1994 sobre los Aspectos de los Derechos de Propiedad Intelectual Relacionados con el Comercio, ADPIC), la inversión global aumentó durante unos cinco años y luego comenzó un descenso continuo. Nuestro argumento es que este declive global se debe atribuir a la erosión progresiva del conocimiento no privatizado disponible.

Gráfica 1

Patentes globales e inversiones globales

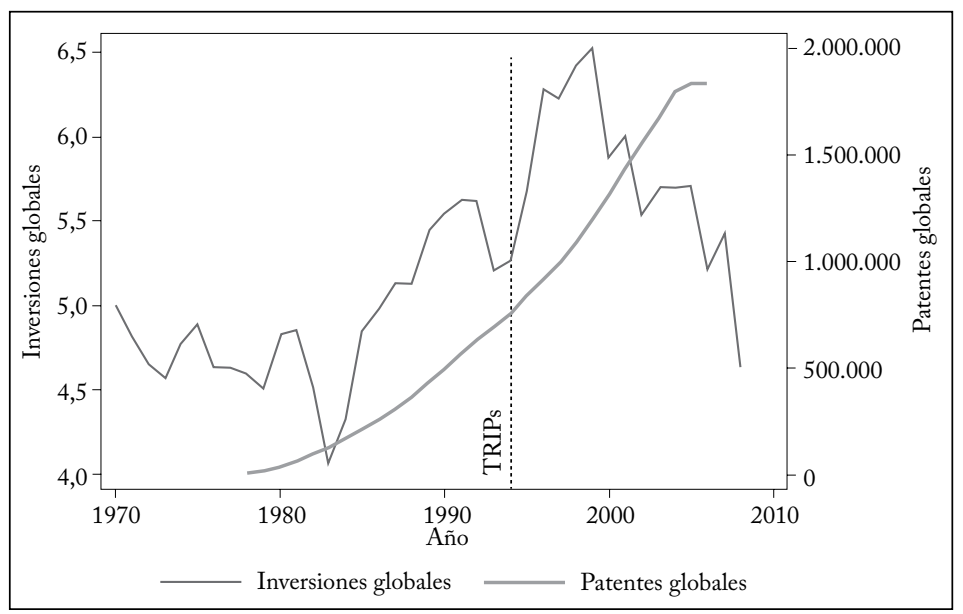

Fuente: Belloc y Pagano (2012).

Así, la desigual distribución del conocimiento es una causa importante de la desigualdad económica general y un freno del crecimiento global. En un libro muy aclamado, Picketty (2014) subraya que una tasa de 
ganancias mayor que la tasa de crecimiento lleva necesariamente a un creciente empobrecimiento relativo de la mayoría de la población. Picketty atribuye el origen de este fenómeno a la excesiva acumulación de capital. Pero una lectura cuidadosa de la evidencia sugiere que una explicación más convincente es la subinversión en bienes reales de capital, característica de las últimas décadas (Rowthorn, 2014). Esta subinversión concuerda con el aumento de la riqueza de las firmas porque este último suele provenir del incremento de sus rentas de monopolio (y también de la tasa de ganancias total de su capital).

Si el poder de monopolio de las firmas aumenta, se verá como un incremento del ingreso del capital, y su valor presente descontado se verá como un aumento de la riqueza (puesto que los derechos sobre las rentas asociadas al poder de mercado se pueden comprar y vender (Stiglitz, 2015, 24).

Aunque hasta ahora descuidada, la privatización del conocimiento puede ser una parte importante de la explicación de la enigmática ocurrencia simultánea de subinversión, acumulación de riqueza, altas ganancias y bajo crecimiento. Cuando gran parte del conocimiento pasa de la esfera pública a la privada es probable que el aumento de la monopolización eleve las ganancias y reduzca el crecimiento. Además, casi por definición, sustraer recursos de conocimiento público aumenta la desigualdad: todos tienen igual derecho de acceso a un bien público. En cambio, la privatización del conocimiento implica que solo el propietario monopólico tiene pleno acceso a él. Por tanto, es probable que el aumento de las rentas (incluidas las que no provienen de la monopolización del conocimiento) ocasione un descenso del crecimiento y un aumento de la desigualdad.

\section{ECONOMÍA POLÍTICA DE LOS VALLADOS DE CONOCIMIENTO}

Como se mencionó de pasada en un párrafo anterior, el punto de inflexión en la protección global de los DPI fue el Acuerdo ADPIC de 1984. Este es el primer acuerdo internacional sobre asuntos de PI que, además de armonizar (hacia arriba) casi todos los aspectos de la protección legal de las creaciones intelectuales a nivel global, prevé un mecanismo de observancia expreso, bajo supervisión de la Organización Mundial del Comercio (oMc). Desde ese acuerdo, otros acuerdos menos generales pero igualmente relevantes han reforzado la protección global de la pi. En Australia, Chile, Perú, en Oriente Medio y otros países se han introducido disposiciones ADPIC plus como parte de la negociación de áreas preferenciales de comercio (APC) con Estados Unidos y la Unión Europea. Asimismo, los convenios entre países en desarrollo (p. ej., entre miembros de la ASEAN) han elevado 
sus estándares y métodos armonizados de PI. Las disposiciones que refuerzan la PI también son comunes en muchos tratados bilaterales de inversión y acuerdos internacionales de inversión (Maskus, 2014).

Las actividades de caza de rentas de las grandes firmas (y dotadas de PI) de los países desarrollados han sido desencadenantes de estos desarrollos. Esto es cierto en particular de las firmas estadounidenses que, al inicio de la Ronda Uruguay de negociaciones, percibían que estaban rezagadas frente a las alemanas y japonesas y ansiaban aumentar las rentas de monopolio y oligopolio que se podían apropiar en los mercados internacionales. Estos intereses privados pueden haberse traducido en captura de negociadores (Lanjouw y Cockburn, 2001).

Sin embargo, los diseñadores de políticas de los países pueden tener incentivos excesivos para fortalecer la protección global de la PI incluso descartando problemas de captura de políticos. Scotchmer (2004) sugiere que la armonización de la protección de la pi lleva a una protección de la pi más amplia que la que se elegiría si las opciones fueran independientes y más fuerte que la que sería óptima desde el punto de vista social. También muestra que, con los requisitos de reciprocidad (trato nacional) incorporados en el Acuerdo ADPIC, los países tienen un incentivo para inclinar la combinación de políticas en favor de la pi a costa del patrocinio público de la investigación y la innovación debido a que este permite internalizar las externalidades de conocimiento transfronterizo.

Más en general, si el conocimiento es un bien común global está expuesto a los problemas usuales que afectan a este tipo de bienes. Aparte de las sutilezas de la economía de los acuerdos de comercio internacionales, es claro que cada país tiene un incentivo para usar el conocimiento público de otros países y privatizar excesivamente el conocimiento que produce, aún más con la protección global armonizada. De ahí que cada país sea impulsado hacia un conjunto de instrumentos de manejo de la pi que aumenta el peso del patentamiento más allá del que sería en una economía cerrada. Este usufructo gratuito nacional del conocimiento común también se puede ver como una forma de competencia desleal, y es sorprendente que la omc permita restricciones al comercio de países que violan la PI de otros países pero permita este tipo de competencia desleal (Pagano, 2014).

Este incentivo basado en el usufructo gratuito para favorecer el conocimiento privatizado frente a la ciencia abierta va de la mano con los grandes cambios que han experimentado los sistemas de investigación pública desde mediados de los noventa. Cuanto más se reconoce que el conocimiento es un ingrediente clave del cre- 
cimiento, más se orientan las universidades y las oIP a satisfacer las necesidades de capacitación e investigación de la economía. Se han hecho reformas para fortalecer e intensificar la colaboración entre el sector público y el privado, para promover el patentamiento y la transferencia de tecnología basada en patentes, así como la creación de oficinas especializadas en transferencia de tecnología (отт), para dirigir los esfuerzos de investigación hacia necesidades sociales específicas mediante la creciente asignación competitiva de fondos (Geuna y Rossi, 2015). Estos desarrollos están cambiando la actitud global de las instituciones de financiación pública de la ciencia y de los mismos investigadores, y afectando profundamente el conjunto de normas asociadas a la ciencia abierta (Dasgupta y David, 1994).

Esto tiende a extender a la investigación pública los efectos de retroalimentación que operan en firmas y países: una vez establecidas las instituciones de PI, los productores de conocimiento (bien sean investigadores, firmas o países) se encuentran en una situación de dilema del prisionero en la que el patentamiento es una estrategia dominante, aunque no patentar maximizaría el bienestar conjunto.

Así, también desde el ángulo de la economía política, las actuales instituciones (internacionales) de la economía del conocimiento parecen incorporar un mecanismo endógeno que tiende a perpetuar su existencia y sus efectos negativos sobre el aprendizaje, el crecimiento y la desigualdad. Y aún más importante, cuando las instituciones de investigación pública antes orientadas a la ciencia abierta son cada vez más activas en el juego de la PI, faltan antídotos endógenos para el creciente estrechamiento del conocimiento público. En la siguiente sección argumentamos que la urgente necesidad de ese antídoto se debería concebir como nueva justificación y fundamento de la política científica, en particular de una política científica global. A falta de tal antídoto, la economía del conocimiento difícilmente podrá cumplir su promesa de propiciar el crecimiento.

\section{NUEVA JUSTIFICACIÓN DE UNA POLÍTICA CIENTÍFICA GLOBAL}

La literatura económica señala la existencia de numerosas justificaciones de la política científica (y tecnológica). En el enfoque neoclásico (Arrow, 1962; Dasgupta y David, 1994), el principal fundamento de la política científica es la existencia de fallas de mercado asociadas al carácter de bien público del conocimiento que constituye la ciencia básica. Mientras que la tecnología se puede apropiar de manera privada mediante DPI, la investigación fundamental genera efectos de 
propagación que motivan la inversión pública en su producción para compensar la falta de incentivos privados.

En contraste, la literatura sobre sistemas o redes de innovación justifica la intervención pública en ciencia y tecnología por fallas del sistema de innovación (ver, p. ej., Freeman, 1995; Lundvall, 2007). Puesto que la innovación depende de las complementariedades y los vínculos entre numerosos recursos y actores, que pueden estar sujetos a problemas de coordinación y de alineación de incentivos, la política pública puede ayudar a resolver estos problemas.

Los autores evolucionistas resaltan un papel adicional de la política científica: la necesidad de difundir el conocimiento y generar diversidad para corregir las consecuencias de sendas evolutivas dependientes de la trayectoria (Nelson y Winter, 1982; Dosi, 1988).

Por último, los exponentes del enfoque basado en el conocimiento, que subrayan el carácter colectivo de la producción, el intercambio y la distribución de conocimientos y la importancia de los procesos de aprendizaje, justifican la política científica y tecnológica por fallas de aprendizaje (cognitivas) (ver Cohendet y Meyer, 2001).

La perspectiva que proponemos en este artículo (y en trabajos anteriores) ofrece una nueva justificación de la política científica (global). Hasta ahora hemos expuesto dos argumentos principales. El primero es que la excesiva privatización del conocimiento debe ser considerada responsable de la reducción y distorsión de las oportunidades de inversión y, en últimas, de obstaculizar el crecimiento y aumentar la desigualdad global. El segundo es que la economía política subyacente a la protección internacional global de la PI y a la inversión en investigación pública tiende a magnificar los efectos de la privatización del conocimiento, dejando al "capitalismo de monopolio intelectual" (Pagano, 2014) sin un mecanismo endógeno para corregir los desequilibrios causados por esta privatización. De estos dos argumentos sacamos tres principales conclusiones para la política científica.

Primera, el carácter (cuasi) público del conocimiento no solo se debería interpretar como una justificación de la necesidad de financiación pública para substituir incentivos privados, enfrentando así una falla de mercado. Una falla aún más perniciosa del sistema se deriva de la excesiva reducción del campo de conocimientos no privatizados, debido a que la propiedad del conocimiento da lugar a dinámicas positivas y negativas que se auto refuerzan y a la reducción general de las oportunidades de inversión comentadas en párrafos anteriores. La ciencia abierta, concebida como el conocimiento científico que 
preserva sus rasgos de bien público, es entonces clave para desbloquear las características de la economía del conocimiento que propician el crecimiento.

Segunda, es necesario ampliar el conjunto de instrumentos de política científica con herramientas que preserven la apertura. Mazzucato (2013) demostró en forma convincente que la inversión pública en ciencia sustenta muchas de las innovaciones más exitosas de nuestra época, de las que se apropió el sector privado. Esto respalda la propuesta de preservar y mejorar la financiación pública de la investigación. Sin embargo, dado el actual marco institucional sesgado en favor de privatizar los resultados de la investigación básica, quizá no baste aumentar la financiación pública de la investigación. En otras palabras, proponemos que el tema de si y cuánta investigación pública se debería financiar no se debe considerar por aparte del tema más fundamental de si se debe alentar la privatización de los resultados de la investigación pública a través de la PI. Las características de la investigación financiada públicamente que mejoran la eficiencia radican en la amplia gama de externalidades que puede propagar en toda la economía. Si faltan estas características, no es claro por qué se debería financiar investigación pública.

Tercera, la dimensión global de la producción de conocimiento y las dinámicas de economía política asociadas se deberían tomar en cuenta explícitamente como fundamento de la política científica. A diferencia de la PI, en el campo de la ciencia abierta no hay organizaciones internacionales que aseguren la armonización de las políticas de patrocinio público para enfrentar los desincentivos de las externalidades transfronterizas (Schotchmer, 2004; Pagano y Rossi, 2009). A pesar de innegables dificultades objetivas, se deberían encontrar maneras de diseñar políticas científicas de dimensión global. La coordinación internacional es urgente y necesaria para asegurar que el principal motor de crecimiento - la producción de conocimiento- no se quede sin el combustible indispensable: el conocimiento científico de acceso gratuito.

Ya se han propuesto algunas medidas posibles a este respecto. Stiglitz (1999) sugirió que por razones de eficiencia y equidad se justificaría que la comunidad internacional "exija el derecho a cobrar por el uso del conocimiento global común”. Una manera de lograr un resultado similar sería exigir una inversión mínima (p. ej., un $3 \%$ del Рів) en ciencia abierta a todos los países miembros de la OMC (Pagano, 2014). Como alternativa, la financiación de instituciones internacionales de conocimiento y de compra de patentes 
respaldadas internacionalmente puede ser parte del conjunto de instrumentos (Pagano y Rossi, 2009). Hay, por cierto, una escasez de soluciones de política creativas en este campo: la búsqueda de maneras de superar los efectos negativos de la excesiva privatización del conocimiento debería ser parte de la agenda de una economía política de la ciencia.

\section{CONCLUSIONES Y PREGUNTAS PARA FUTURA INVESTIGACIÓN}

En este artículo mostramos que existe un nuevo fundamento para una política científica global: la necesidad de contrarrestar las nefastas consecuencias de la excesiva privatización del conocimiento. La promesa de la economía del conocimiento de propiciar el crecimiento quizá nunca se cumpla debido a su tendencia endógena a esta excesiva privatización. La exclusión y el bloqueo excesivos en el uso de los recursos de conocimiento ya han manifestado sus efectos no solo en los patrones de especialización de la producción internacional y de crecimiento desigual, sino también en la reducción del potencial de crecimiento de los países que poseen las mejores dotaciones de capital intelectual privatizado. Además, la economía política de la protección global de la PI y de la inversión en investigación pública sugiere que esta reducción de las oportunidades de inversión y de crecimiento no encuentra antídotos fáciles.

También sugiere un conjunto de nuevas preguntas para una economía política de la ciencia. Primera, se debe profundizar el estudio de los efectos de retroalimentación virtuosos y viciosos entre la distribución de activos intelectuales y la inversión en aprendizaje y conocimiento, para identificar maneras de romper los círculos viciosos y desbloquear el potencial de la economía del conocimiento. Segunda, se necesita más investigación para entender plenamente los efectos globales del cambio de actitudes y prácticas de la investigación financiada públicamente, que se alejan cada vez más de las normas de ciencia abierta para acoger normas de "ciencia cerrada". ¿Hasta qué punto los movimientos por el libre acceso y las políticas que promueven algunas universidades y grupos de investigación pueden ser una solución apropiada? ¿Qué alternativas están disponibles? Tercera, una pregunta más esencial se refiere a la identificación de maneras de superar la arraigada resistencia a idear soluciones globales para producir ciencia abierta y, más en general, de conocimiento públicamente disponible. Estas preguntas no agotan el conjunto de temas relevantes para la agenda de investigación de una economía política nueva y global de la política científica. Pensamos, sin embargo, que son un punto de 
partida necesario si se quieren evitar más crisis y depresiones de la economía del conocimiento.

\section{REFERENCIAS BIBLIOGRÁFICAS}

1. Abramovitz, M. "The welfare interpretation of secular trends in national income and product”, M. Abramovitz et al., eds., The allocation of economic resources: Essays in honor of Bernard Francis Haley, Stanford, Ca., Stanford University Press, 1959.

2. Arrow, K. J. “Technical information and industrial structure”, Industrial and Corporate Change 5, 2, 1996, pp. 645-652.

3. Azoulay, P.; W. Ding y T. Stuart. "The impact of academic patenting on the rate, quality and direction of (public) research output", Journal of Industrial Economics 57, 4, 2009, pp. 637-676.

4. Belloc, F. y U. Pagano. "Knowledge enclosures, forced specialization and investment crisis", European Journal of Comparative Economics 9, 3, 2012, pp. 445-483.

5. Benkler, Y. "Intellectual property and the organization of information production", International Review of Law and Economics 22, 2002, pp. 81-107.

6. Bessen, J. E. y M. J. Meurer. Patent failure: How judges, bureaucrats, and lawyers put innovators at risk, Princeton, Princeton University Press, 2008.

7. Boldrin, M. y D. K. Levine. Against intellectual monopoly, Cambridge, Cambridge University Press, 2008.

8. Campbell, E. G. et al. "Data withholding in academic genetics: Evidence from a national survey", jama 287, 4, 2002, pp. 473-480.

9. Cockburn, I.; M. MacGarvie y E. Mueller. "Patent thickets, licensing and innovative performance", Industrial and Corporate Change 19, 3, 2010, pp. 899-925.

10. Cohen W.; R. Nelson y J. Walsh. "Protecting their intellectual assets: Appropriability conditions and why U.S. manufacturing firms patent (or not)", NBER working paper 7552, 2000.

11. Cohendet, P. y F. Meyer-K. "The theoretical and policy implications of knowledge codification", Research Policy 30, 9, 2001, pp. 1563-1591.

12. Dosi, G. "Sources, procedures and microeconomic effects of innovation”, Journal of Economic Literature 26, 3, 1988, pp. 1120-1171.

13. Duguet, E. e I. Kabla. "Appropriation strategy and the motivations to use the patent system: An econometric analysis at the firm level in French manufacturing" 1998, Annales d'Économie et de Statistique 49-50, 1998, pp. 289-327.

14. Franzoni, C. y G. Scellato. "The grace period in international patent law and its effect on the timing of disclosure", Research Policy 39, 2, 2010, pp. 200-2013.

15. Franzoni, C.y G. Scellato. "Academic patenting and the consequences for scientific research", The Australian Economic Review 44, 1, 2011, pp. 95-101.

16. Freeman, C. "The 'national system of innovation' in historical perspective”, Cambridge Journal of Economics 19, 1, 1995, pp. 5-24. 
17. Geuna, A. y F. Rossi. The university and the economy. Pathways to growth and economic development, Cheltenham, Edward Elgar, 2015.

18. Geuna, A. y L. Nesta. "University patenting and its effects on academic research: The emerging European evidence", Research Policy 35, 6, 2006, pp. 790-807.

19. Hall, B. H. y R. H. Ziedonis. "The patent paradox revisited: An empirical study of patenting in the U.S. semiconductor industry 1979-1995", rand Journal of Economics 32, 1, 2001, pp. 101-128.

20. Hart, O. Firms, contracts and financial structure, Oxford, Oxford University Press, 1995.

21. Heller, M. y R. Eisenberg. "Can patents deter innovation? The anticommons in biomedical research", Science 280, 5364, 1998, pp. 698-701.

22. Jaffe A. B. y J. Lerner. Innovation and its discontents: How our broken patent system is endangering innovation and progress, and what to do about it, Princeton, Princeton University Press, 2004.

23. Lanjouw, J. O. y I. M. Cockburn. "New pills for poor people? Evidence after GATT", World Development 29, 2, 2001, pp. 265-249.

24. Lemley, M. A. "Property, intellectual property, and free riding", Texas Law Review 83, 2005, pp. 1031-1069.

25. Lerner, J. "Patenting in the shadow of competitors", Journal of Law and Economics 38, 2, 1995, pp. 463-490.

26. Levin, R. et al. "Appropriating the returns from industrial R\&D”, Brookings Papers on Economic Activity 3, 1987.

27. Lissoni, F. et al. "Academic patenting in Europe: New evidence from the KeIns database", Research Evaluation 17, 2, 2008, pp. 87-102.

28. Lundvall, B.-Å. "National innovation systems - Analytical concept and development tool", Industry and innovation 14, 1, 2007, pp. 95-119.

29. Mansfield E. "Patents and innovation: An empirical study", Management Science 32, 2, 1986, pp. 173-181.

30. Maskus, K. "The new globalization of intellectual property rights: What's new this time?", Australian Economic History Review 54, 3, 2014, pp. 262-284.

31. Mazzucato, M. The entrepreneurial State: Debunking public vs. private sector myths, Londres, Anthem Press, 2013.

32. Merges R. y R. Nelson. "On the complex economics of patent scope", Columbia Law Review 90, 4, 1990, pp. 839-916.

33. Murray, F. et al. "Of mice and academics: Examining the effect of openness on innovation", NBER working paper 14819, 2009.

34. Noel, M. y M. Schankerman. "Strategic patenting and software innovation", Journal of Industrial Economics 61, 3, 2013, pp. 481-520.

35. Pagano U. "The crisis of intellectual monopoly capitalism", Cambridge Journal of Economics 38, 6, 2014, pp. 1409-1429.

36. Pagano U. y M. A. Rossi. "Incomplete contracts, intellectual property and institutional complementarities", European Journal of Law and Economics 18, 1, 2004, pp. 55-76.

37. Pagano U. y M. A. Rossi. "The crash of the knowledge economy", Cambridge Journal of Economics 33, 4, 2009, pp. 665-683. 
38. Pagano U. y M. A. Rossi. "Property rights in the knowledge economy: An explanation of the crisis", E. Brancaccio y G. Fontana, eds., The global economic crisis, Londres, Routledge, 2011, pp. 284-297.

39. Partha, D. y P. A. David. "Toward a new economics of science", Research Policy 23, 5, 1994, pp. 487-521.

40. Piketty, T. Capital in the Twenty-First Century, Cambridge, Mass., Harvard University Press, 2014.

41. Reitzig, M.; J. Henkel y F. Schneider. "Collateral damage for R\&D manufacturers: How patent sharks operate in markets for technology", Industrial and Corporate Change 19, 3, 2010, pp. 947-967.

42. Rowthorn, R. "A note on Piketty's Capital in the Twenty-First Century", Cambridge Journal of Economics 38, 5, 2014, pp. 1275-1284.

43. Samuelson, P. "Enriching discourse on public domains", Duke Law Journal 55, 2006, pp. 783-834.

44. Schumpeter, J. A. The theory of economic development: An inquiry into profits, capital, credit, interest, and the business cycle [1934], New Brunswick, NJ, Transaction Publishers, 1983.

45. Schumpeter, J. A. Capitalism, socialism, and democracy, Nueva York, Harper and Brothers, 1942.

46. Scotchmer, S. "Standing on the shoulders of giants: Cumulative research and the patent law", Journal of Economic Perspectives 5, 1, 1991, pp. 29-41.

47. Scotchmer, S. "The political economy of intellectual property treaties", Journal of Law, Economics, and Organization 20, 2, 2004, pp. 415-437.

48. Shapiro, C. "Navigating the patent thicket: Cross licenses, patent pools, and standard setting”, A. Jaffe, J. Lerner y S. Stern, eds., Innovation policy and the economy, vol. 1, Cambridge, Mass., Mit Press, 2001, pp. 119-150.

49. Solow, R. M. "Investment and technical progress”, K. Arrow, S. Karlin y P. Suppes, eds., Mathematical methods in the social sciences [1959], Stanford, Ca., Stanford University Press, 1960, pp. 89-104.

50. Stiglitz J. E. "New theoretical perspectives on the distribution of income and wealth among individuals", NBER working paper 21189, 2015.

51. Stiglitz, J. E. "Knowledge as a global public good", 1999, [http:// p2pfoundation.net/Knowledge_as_a_Global_Public_Good].

52. Stokes, D. Pasteur's quadrant: Basic science and technological innovation, Washington, Brookings Institution Press, 1997.

53. Thursby, J. y M. Thursby. "University licensing”, Oxford Review of Economic Policy 23, 4, 2007, pp. 620-639.

54. Walsh, J. P.; W. M. Cohen y C. Cho. "Where excludability matters: Material versus intellectual property in academic biomedical research", Research Policy 36, 8, 2007, pp. 1184-1203. 